\title{
Developing discriminate model and comparative analysis of differentially expressed genes and pathways for bloodstream samples of diabetes mellitus type 2
}

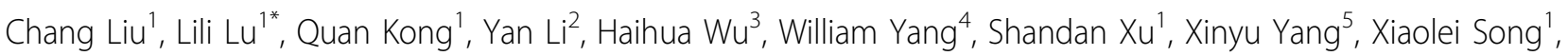
Jack Y Yang ${ }^{6,7}$, Mary Qu Yang ${ }^{8,9}$, Youping Deng ${ }^{1,2^{*}}$

From 2014 International Conference on Bioinformatics and Computational Biology

Las Vegas, NV, USA. 21-24 July 2014

\begin{abstract}
Background: Diabetes mellitus of type 2 (T2D), also known as noninsulin-dependent diabetes mellitus (NIDDM) or adult-onset diabetes, is a common disease. It is estimated that more than 300 million people worldwide suffer from T2D. In this study, we investigated the T2D, pre-diabetic and healthy human (no diabetes) bloodstream samples using genomic, genealogical, and phonemic information. We identified differentially expressed genes and pathways. The study has provided deeper insights into the development of T2D, and provided useful information for further effective prevention and treatment of the disease.

Results: A total of 142 bloodstream samples were collected, including 47 healthy humans, 22 pre-diabetic and 73 T2D patients. Whole genome scale gene expression profiles were obtained using the Agilent Oligo chips that contain over 20,000 human genes. We identified 79 significantly differentially expressed genes that have fold change $\geq 2$. We mapped those genes and pinpointed locations of those genes on human chromosomes. Amongst them, 3 genes were not mapped well on the human genome, but the rest of 76 differentially expressed genes were well mapped on the human genome. We found that most abundant differentially expressed genes are on chromosome one, which contains 9 of those genes, followed by chromosome two that contains 7 of the 76 differentially expressed genes. We performed gene ontology (GO) functional analysis of those 79 differentially expressed genes and found that genes involve in the regulation of cell proliferation were among most common pathways related to T2D. The expression of the 79 genes was combined with clinical information that includes age, sex, and race to construct an optimal discriminant model. The overall performance of the model reached $95.1 \%$ accuracy, with $91.5 \%$ accuracy on identifying healthy humans, $100 \%$ accuracy on pre-diabetic patients and $95.9 \%$ accuract on T2D patients. The higher performance on identifying pre-diabetic patients was resulted from more significant changes of gene expressions among this particular group of humans, which implicated that patients were having profound genetic changes towards disease development.
\end{abstract}

Conclusion: Differentially expressed genes were distributed across chromosomes, and are more abundant on chromosomes 1 and 2 than the rest of the human genome. We found that regulation of cell proliferation actually plays an important role in the T2D disease development. The predictive model developed in this study has utilized

\footnotetext{
* Correspondence: Iulili@wust.edu.cn; youping_deng@rush.edu

'Wuhan University of Science and Technology, Wuhan, China

Full list of author information is available at the end of the article
}

(c) 2014 Liu et al: licensee BioMed Central Ltd. This is an Open Access article distributed under the terms of the Creative Commons 


\section{Introduction}

Diabetes mellitus of type 2 (T2D) is among 10 most common diseases. According to World Health Organization (WHO), it is estimated that 347 million people suffer from type 2 diabetes. T2D is usually considered not reversible, but if not controlled well, it will eventually lead to fatal complications. However, earlier diagnosis of diabetes requires preventative screening and regular healthcare monitoring, which are not always provided in many countries. Therefore, lower income countries have higher death rates from diabetes. WHO estimated that diabetes deaths will be doubled by the year of 2030 . Unfortunately about half of diabetes patients do not realize that they have the disease [1]. In United States of America, diabetes commonly occurs in all ethnic population, and is the seventh most common cause of death. According to American Diabetes Association, the prevalence of the disease is now climbing towards $10 \%$ and 30 million people. T2D is also an age related disease. The prevalence in seniors is more than a quarter of the entire population. Because T2D is related to lifestyles, especially foods and exercises, the disease has been and will be consistently increasing and has been estimated to reach one third of the population by 2050 [2]. While type 1 diabetes mellitus is known as juvenile diabetes with strong genetic dispositions or viral involvement, type 2 diabetes is known genetically diverse and contribute to more than $90 \%$ of all diabetes [3]. All diabetes can lead to not only long-term but also lethal complications, including cardiovascular, retina, nerve system complication, chronic renal failure, and greater susceptibility of infection. Because T2D is so popular, it has led to the increasing death rate as well as social and economic burdens. While T2D has been known as a genetically complex and multifactorial disease with impaired glucose regulation, such as impaired fasting glucose (IFG) and impaired glucose tolerance (IGT), it is a gradually developing disease and commonly considered as irreversible at our current treatment capabilities. T2D often proceed gradually with aging and can be worsened by many factors, such as hypertension, high cholesterol, lacking of exercise, genetic disposition and family history of diabetes [4-7]. Obesity and immune/inflammatory issues can contribute to the disease $[8,9]$. The changes of modern lifestyles in humans have been considered an important factor in the ever-increasing T2D occurrences.

While so far no effective treatment can virtually cure diabetes, significant research progresses have been made in understanding the genetic changes in the development of T2D, especially the mechanisms of gene regulation. New research has led to better prevention of the development of T2D and effective identification of drug targets for blocking or even reversing the disease development [10]. Frayling from the UK's Peninsula College of Medicine and Dentistry found that Single Nucleotide Polymorphism in fat mass and obesity associated gene (FTO) has a strong association with the risk of T2D [11]. Zhao's research showed that analysis of combined gene expression and lipid profiles helped to identify the pathogenesis of T2D [12]. As our research has been unfolded to the study of differential expression of genes and regulatory mechanisms of the genes in diabetes mellitus, we have made significant progresses in finding specific genes and the specific pathways to be targeted by drugs for the purposes of preventing and inversing the disease development.

Pre-diabetic or early stage T2D patients usually do not have any noticeable symptoms and are not diagnosable without blood analysis. Clinical diagnosis of diabetes includes fasting plasma glucose test [13], hemoglobin A1c test, glucose tolerance test, and clinical screening during physical checkup, but all rely on laboratory blood analysis [14]. Although blood analysis does not provide any genetic, genomic or pathological information about the disease, such information can be useful in assessing the stage, subtype, prognosis, damage and impact of the disease. New research efforts include differential gene expression profiling and genome-wide association studies (GWAS) [15-19] have been made. Yet, the molecular mechanisms of disease are potentially heterogeneous while the limited availability of samples for genome-wide association studies almost prevented effective population genetics analysis and sub-type identification of the disease. In this paper, we assessed and analyzed a number of patients and healthy humans using genomic information including peripheral white blood cell gene expression profile (GEP), and phonemic information including age, gender, and race. Our past studies found that phonemic factors have little influence on GEP. Hierarchical clustering and principal component analysis (PCA) showed that GEP were not directly related to the phonemic factors including gender, blood sugar level, age, and race. However, race and gender are not randomly distributed in the clustering analysis, which implicated that they had potential relevancy with GEP [20]. Therefore, we use age, gender, race, and 79 significant genes as parameters to derive 
the discriminant model. The model was used successfully to classify the samples of different disease stages respectively with high performance and accuracy. This has led to find molecular mechanisms and genetic diversity for identifying sub-types and pathogenesis of type 2 diabetes mellitus.

\section{Materials and methods}

\section{Research objectives, and laboratory and clinical data}

Based on our previous research, we now aim to identify characteristic genes and pathways in diabetes. The criteria for the diagnosis of T2D were based on the American Diabetes Association (ADA) [21] guidelines in accordance with the symptoms in diabetes. The diagnostic criteria are positively correlated with body mass index (BMI) and fasting blood glucose level $>126 \mathrm{mg} / \mathrm{dl}$, or $2 \mathrm{H}$ blood glucose level $>200 \mathrm{mg} / \mathrm{dl}$ in the oral glucose tolerance test. A total of 142 bloodstream samples were collected, including 47 people from a healthy control group, 22 pre-diabetic, and 73 T2D patients. The experiments were carried out and analyzed using comprehensive information that includes age, gender, race, and GEP. Tougaloo College in Mississippi provided data, and the research was approved by the Institutional Review Board of Tougaloo.

\section{RNA isolation}

Firstly, Total RNA from $8-10 \mathrm{ml}$ of peripheral blood white cells was extracted according to the manufacturer's instructions with LeukoLock ${ }^{\mathrm{TM}}$ general RNA system (Anbion Inc, Austen, Texas, USA). Then the content of the RNA was detected and separated by using Nanodrop spectrophotometer and Agilent 2100 Bioanalyzer (Agilent Technologies, Santa Clara, California, USA). All protocols have been carefully assured and all RNAs have been carefully inspected to ensure that RNAs were not degenerated.

\section{Microarray experiments}

All standard protocols and instructions on handling RNA have been carefully followed. A total of $500 \mathrm{ng}$ of RNA was amplified and labeled by Agilent Low RNA Input Fluorescent Linear Amplification. 850 ng of Cy5(universal control) and Cy3-labeled (sample) cRNA were mixed and dispersed by the Agilent In Situ Hybridization Kit for every two color array. According to the Agilent 60-mer oligo microarray, hybridizations were put forward for 17 hours in rotating hybridization oven first, and then an Agilent Scanner (G2565AA, Agilent Technologies, Wilmington, Delaware, USA) was used to wash and scan them. Finally, quality control analysis was performed and the Agilent Feature Extraction software (v.9.5.3.1) was used to handle the information from the chips and correction of background noises from default parameters.

\section{Microarray data analysis}

Gene chip data was analyzed using GeneSpring 10.0 and quality control was conducted with Pearson correlation coefficients between each sample of the experiment and others pair-wisely. Samples showing less than $80 \%$ of correlation with other samples were excluded for further analysis. Scanning probe intensity of less than 5 was directly converted into 5 . All probe values using the chip in the 5000 percentile were standardized as perchip (inner) data. Each gene was standardized using the median value of one gene in all of the samples. The probe characteristics were screened by markers while "Occupying" or "Absence" of the symbol could be used to define the Aglient properties. The Database for Annotation, Visualization and Integrated Discovery (DAVID, http://david.abcc.ncifcrf.gov/) online analysis tool was used to analyze chromosomal localization and function of differentially expressed genes.

\section{Statistical analyses \\ Analysis of differentially expressed genes}

GeneSpring GX 10.0 (Agilent Technologies, Santa Clara, California, USA) software was used for gene expression analysis. To standardize the data using the method of Lowess [22], each chip used the $50 \%$ as the base, and each gene was standardized by a median reference. The standardized sample data was entered into the GeneSpring GX software. Firstly, quality controls were performed to select the data and samples of the inserted microarray data. Secondly, all the samples were divided into 3 groups: the normal group (ND), pre-diabetic group (PD), and T2D group (D). Thirdly, matched pairwise analysis was conducted and groups were compared pair-wisely. Finally, less significant genes were filtered out. The selection threshold was set as: False discovery rate $(\mathrm{FDR})=5 \%, \mathrm{p}<0.05, \mid$ fold change $\mid \geq 2$.

\section{Discriminate analysis}

Fisher's exact test was used as a discriminant method and a discriminant model was built by all parameters in the experimental group. Statistical software package SPSS 16.0 was used. All samples were finally classified by the discriminant model.

\section{Results}

\section{Quality control on samples and entities}

We performed quality control analysis. Figure 1 showed that the gene chip quality was well controlled and acceptable. The correlation coefficients were greater than 0.9. The correlation plot shows the Pearson correlation coefficient for each pair of array and displays in visual form as a heatmap. The correlation coefficient is a number between 0 and 1 . If there is no relationship between the samples, the correlation coefficient is zero or very low, while high correlation gives a coefficient 


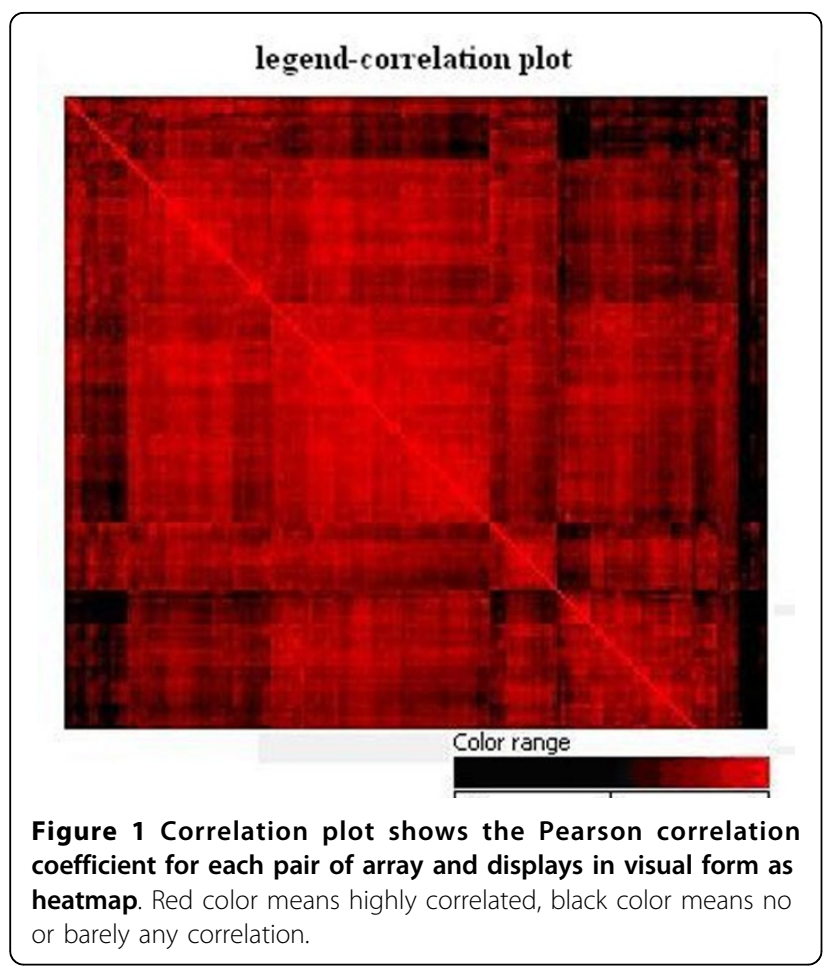

value close to and up to1.0 (Figure 1). Thus, it appears the higher the correlation coefficient, the better quality of the data.

\section{Hierarchical clustering}

Hierarchical clustering analysis was performed on all samples of diabetes, pre-diabetes, and normal human groups as shown in Figure 2. PD group and the other two groups are clearly separated. The results showed that the gene expression levels of PD group from GEP were relatively most significant compared to the other two groups (Figure 2).

\section{Differential genes}

We identified 79 significantly differentially expressed genes with fold change $\geq 2.0$. In the differentially expressed gene analysis between the pre-diabetes, and T2D groups, there are 24 genes with fold change $\geq 2.0$, among which 7 genes were up-regulated and 14 genes were down-regulated. In the comparison between prediabetes and normal human groups, there are 74 genes with fold change $\geq 2.0$, among which 20 genes were upregulated and 14 genes were down-regulated. A number of genes were expressed differentially among all groups. For example TFEB gene was not only significant in the T2D and pre-diabetes comparison but also in the healthy and pre-diabetes comparison, with 2.76 and 3.88 times in difference in each pairs of comparisons respectively.

\section{Chromosome location of differentially expressed genes}

We performed bioinformatics analysis, and found 79 differentially expressed genes with more than 2 fold changes. Locations of 3 of the79 genes are not known and the other 76 differentially expressed genes were distributed across different chromosomes. Chromosome 1 contains 9 of them (11.3\%); and chromosome 2 contains 7 of them $(8.86 \%)$. These two chromosomes are most abundant in significant genes. No differentially expressed genes were found in chromosomes 4, 9, 20, and the sex $Y$ chromosome. It is reasonable that T2D does not have obvious gender difference. 57 genes were located in the long arm of a chromosome, accounting for $72.2 \%$ in total; 19 genes were located in the short arm of a chromosome, accounting for $24.1 \%$. Although chromosome 1 is the longest chromosome in a human genome, differentially expressed genes are still more abundant relatively on chromosomes 1 and 2.

\section{Functional classifications of differentially expressed genes} We performed gene ontology (GO) analysis and classified differentially expressed genes by biological pathways. 11 significant genes were found in the regulation of cell proliferation process, 5 genes were found in taxis process, 5 genes were found in chemotaxis process, 7 genes were found in positive regulation of cell proliferation process, 3 genes existed in sperm motility process and T2D may impact on male sexual functionality. We found 6 significant genes in the localization of cell process, 6 genes in cell motility process, 7 genes in cell motion process, 3 genes in rho protein signal transduction process, 3 genes in rho protein signal transduction process, 5 genes in epithelium development process, 8 genes in cell adhesion process, 8 genes in biological adhesion process, 5 genes in locomotory behavior process, 3 genes in cellular defense response process which implicate that $\mathrm{T} 2 \mathrm{D}$ may impact cellular function. For examples, increased B-cell proliferation has been known in pre-diabetes and implicates that the pancreas may have loosen hormone secretion function. Impaired neutrophil chemotaxis has been known in diabetic patients. Furthermore we found 6 genes in behavior process, 13 genes in cell surface receptor linked signal transduction process, 4 genes in tissue morphogenesis process, 5 genes in negative regulation of cell proliferation process, 4 genes in tube development process, and 3 genes existed in morphogenesis of an epithelium process. Results are summarized in Table 1. The Gene Ontology analysis has provided deeper insights into the molecular mechanisms of T2D that can help the identification of drug targets in blocking the pathways in the disease development.

\section{Discriminate analysis}

A discriminant model was built by 82 parameters (V2V83) from the 142 samples, and V60 was eliminated in 


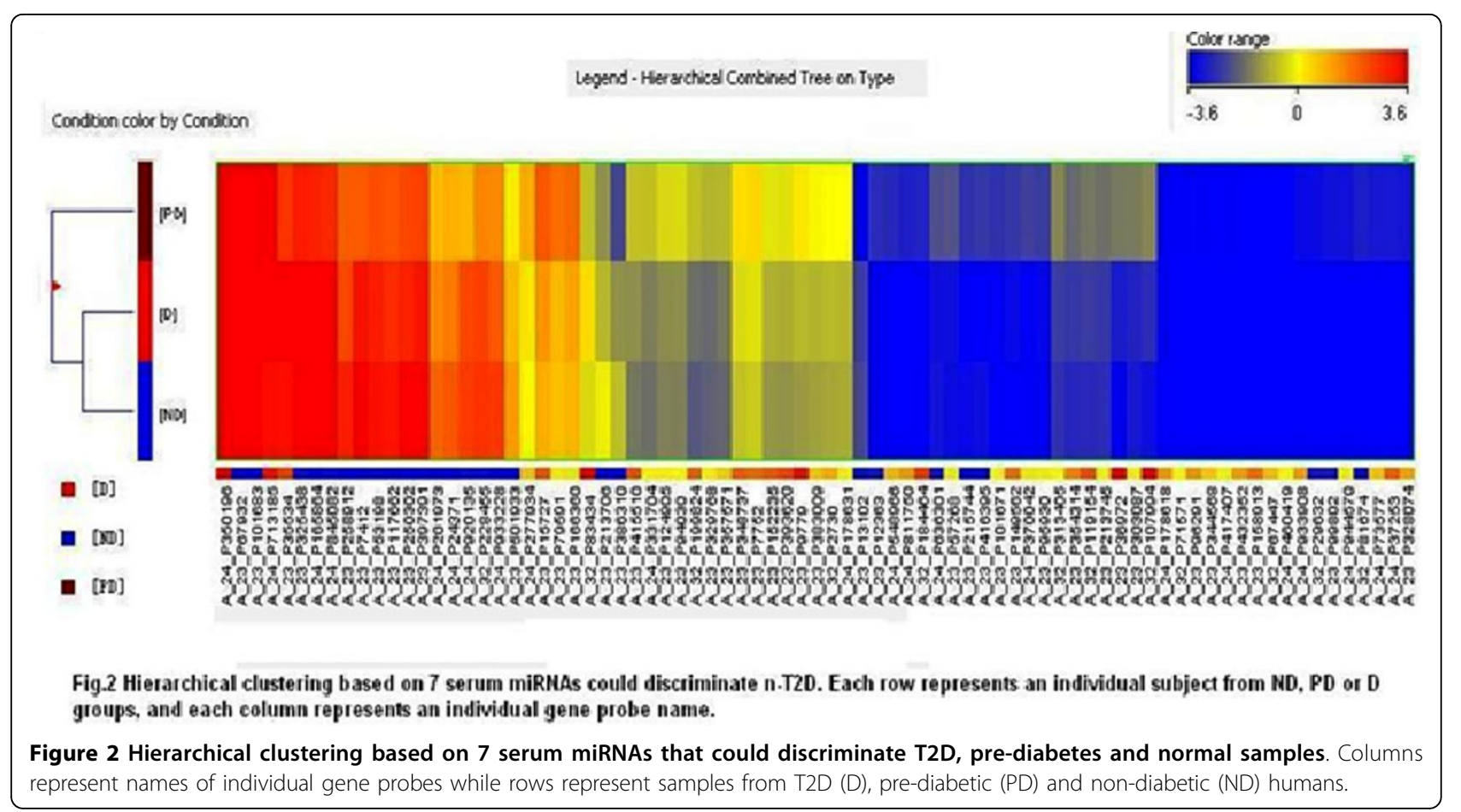

the analysis process. Two typical discrimination functions (Function1, Function2) were extracted, among which Function 1 explained $74.4 \%$ of all variations and Function2 explained the rest $25.6 \%$. Testing results showed a p-value $=0.000$ from Function 1 through Function2, which meant the discriminate function has the greatest statistical significance. The functional expressions of discriminate functions were analyzed.

Table 1 Classification of Biological Process Categories Based on Gene Ontology (GO) analysis

\begin{tabular}{|c|c|c|c|}
\hline Biological Process & Genes & P-value & Count \\
\hline Regulation of cell proliferation & $\begin{array}{l}\text { LAMB1, ALDH1A2, GPNMB, PTN, IL8RB, CXADR, CTTNBP2, PRAME, IL4, IGFBP5, } \\
\text { LAMA1, CXADR }\end{array}$ & 0 & 11 \\
\hline Taxis & CCR3, IL8RB, CXCL14, IL4, IL8RA & 0.002 & 5 \\
\hline Chemotaxis & CCR3, IL8RB, CXCL14, IL4, IL8RA & 0.002 & 5 \\
\hline Postive regulation of cell proliferation & LAMB1, ALDH1A2, PTN, IL8RB, PRAME, IL4, LAMA1 & 0.003 & 7 \\
\hline Sperm motility & LAMA1, APOB, CTTNBP2 & 0.004 & 3 \\
\hline Localization of cell & ROPN1B, APOB, NR2F1, IL8RB, CTTNBP2, LAMA1 & 0.004 & 6 \\
\hline Cell motility & ROPN1B, APOB, NR2F1, IL8RB, CTTNBP2, LAMA1 & 0.004 & 6 \\
\hline Cell motion & ROPN1B, APOB, NR2F1, IL8RB, SEMA6A, CTTNBP2, LAMA1 & 0.006 & 7 \\
\hline Rho protein signal transduction & ROPN1B, COL1A2, ARHGAP29 & 0.008 & 3 \\
\hline Epithelium development & AHNAK, ALDH1A2, ZIC2, NEUROG3, LAMA1 & 0.008 & 5 \\
\hline Cell adhesion & LAMB1, ROPN1B, CCR3, FLRT2, GPNMB, BCAN, CXADR, LAMA1, CXADR & 0.011 & 8 \\
\hline Biological adhesion & LAMB1, ROPN1B, CCR3, FLRT2, GPNMB, BCAN, CXADR, LAMA1, CXADR & 0.011 & 8 \\
\hline Locomotory behavior & CCR3, IL8RB, CXCL14, IL4, IL8RA & 0.016 & 5 \\
\hline Cellular defense response & CCR3, IL8RB, IL4 & 0.02 & 3 \\
\hline Behavior & CCR3, PTN, IL8RB, CXCL14, IL4, IL8RA & 0.025 & 6 \\
\hline $\begin{array}{l}\text { Cell surface receptor linked signal } \\
\text { transduction }\end{array}$ & $\begin{array}{l}\text { P2RY14, CCR3, COL1A2, SEMA6A, IL8RB, PTN, IL8RA, LAMA1, FFAR2, ROR1, GPR161, } \\
\text { HRH4, STC2 }\end{array}$ & 0.026 & 13 \\
\hline Tissue morphogenesis & ALDH1A2, COL1A2, ZIC2, LAMA1 & 0.026 & 4 \\
\hline Negative regulation of cell proliferation & ALDH1A2, GPNMB, CXADR, CTTNBP2, IGFBP5, CXADR & 0.039 & 5 \\
\hline Tube development & ALDH1A2, SALL1, CTTNBP2, ZIC2 & 0.043 & 4 \\
\hline Morphogenesis of an epithelium & ALDH1A2, ZIC2, LAMA1 & 0.05 & 3 \\
\hline
\end{tabular}


The performance of classification results from the discriminant model derived by principles of back substitution was high. Results showed that $95.1 \%$ samples were correctly classified overall, and groups of pre-diabetes were all correctly predicted. The accuracy rate of T2D group was $95.9 \%$ and the accuracy rate of healthy group was $91.5 \%$. The results are summarized in Table 2 . Figure 3 presented a graph of scattered discriminant scores. It can be seen from the Figures 1, 2, 3 that the model can distinguish disease group from health group, especially the distinctions between pre-diabetes group and others were particularly obvious. Therefore, the model built in this study can provide useful information for early biomarker identification of the disease.

\section{Discussion}

While Grayson et al. [23] published an article about the study of human peripheral blood gene chips, their research only showed that the difference of gene expression in T2D played an important role in signal transduction of $\mathrm{T}$ cell activation, but the number of samples used in their study (only six cases) was limited, and their samples did not include racial information. When Lei Kong et. al. [10] discussed the significance of seven microRNA in the serum (miR-9, miR-29a, miR-30d, miR34a, miR124a, miR146a, and miR375) that are related to diabetes, their samples were 56 healthy controls, 18 newly diagnosed T2D patients (n-T2D), and 19 pre-diabetic patients with known susceptibilities (s-NGT). Canonical discriminant analysis results confirmed $70.6 \%$ of n-T2D samples $(12 / 17)$, while the samples of the s-NGT and pre-diabetic could not be distinguished from each other. Rui WangSattler et al [24] quantified 140 metabolites of fasting serum samples of 4297 and confirmed candidate biomarkers for pre-diabetes using metabolomic methods to identify three metabolites [glycine, lysophosphatidylcholine (LPC) and acetyl] for prediction of IGT and T2D. Wang et al. [25] studied the samples of 189 T2D and showed that the increasing content of a small group of essential amino acids [leucine (Leu), valine (Val), isoleucine (Ile)] and aromatic amino acids [phenylalanine (Phe), tyrosine (Tyr)] in serum are associated with risk of T2D by five-fold increment. Our study provides complementary insights into the mechanism of T2D and useful

Table 2 Classification Results

\begin{tabular}{|c|c|c|c|c|c|c|}
\hline & & \multirow[b]{2}{*}{ VAR00001 } & \multicolumn{3}{|c|}{ Predicted Group Membership } & \multirow[b]{2}{*}{ Total } \\
\hline & & & D & & PD & \\
\hline Original & Count & $\mathrm{D}$ & $70(95.9 \%)$ & $3(4.1 \%)$ & $0(0 \%)$ & 73 \\
\hline \multirow{2}{*}{\multicolumn{2}{|c|}{$\%$}} & ND & $4(8.5 \%)$ & $43(91.5 \%)$ & $0(0 \%)$ & 47 \\
\hline & & PD & $0(0 \%)$ & $0(0 \%)$ & $22(100 \%)$ & 22 \\
\hline
\end{tabular}

a. $95.1 \%$ of original grouped cases correctly classified

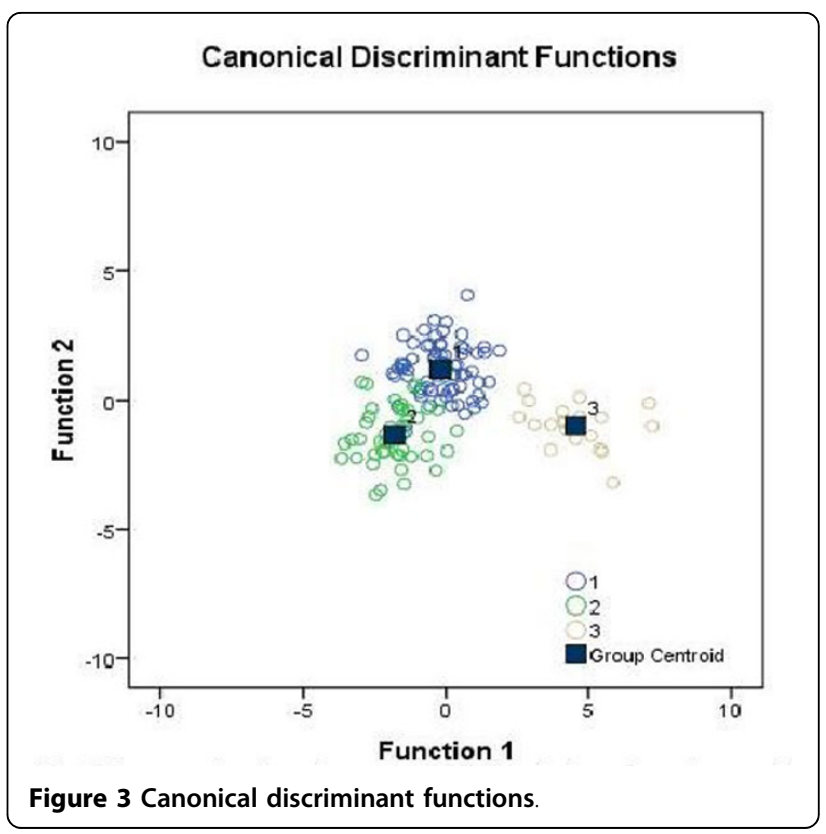

information for better prevention and treatment of the disease and effective identification of drug targets.

\section{Conclusion}

This study identified 79 significant genes with more than 2 -fold changes in differentially expressed genes using bioinformatics approaches. Differentially expressed genes were mainly distributed in chromosomes $1,2,3,5$, and 7 , with more abundance in chromosomes 1 and 2. According to Gene Ontology and gene functional analysis, genes which belong to the regulation of cell proliferation were very significant and played important roles in the pathogenesis of T2D. Many genes have multiple functions. For instance, insulin receptor is involved in diabetes and also plays a role in cell proliferation and cancer. Diabetes is a disorder of metabolic syndrome, which will also induce cell proliferation changes on some tissues. T2D patients may have compromised cellular function in absorbing bloodstream sugar, it is reasonable to have elevated gene expression relating to cell proliferation pathways. This study discussed feasibility of combined molecular and bioinformatics methods to distinguish normal humans, pre-diabetic, and T2D effectively. We have analyzed 142 blood samples, including the healthy control group of 47 people, 22 pre-diabetic, and 73 T2D patients. By comparing the gene chip spectrum of these samples, T2D biomarkers can be implicated from the 79 significant genes. Discriminant analysis model showed that combination of 79 genes with three phonemic factors could effectively distinguish healthy human, pre-diabetic, and T2D patients. The results showed that $95.1 \%$ of the samples were correctly classified, amongst which $100 \%$ was 
acheived in predicting pre-diabetic samples, $95.9 \%$ accuracy was achieved in T2D group, and $91.5 \%$ accuracy in healthy human group. The research provided a combined molecular and pedigree analytic method that could potentially lead to an effective screening tool for identifying overall health or illness of humans and prediction of the prognosis of the disease development. The results also showed that 79 genes are significant in diabetes, and these 79 differentially expressed genes have revealed deeper molecular mechanisms of the disease. The research has also led to effective pathway and drug target identification, treatment planning and future therapeutic strategies. In addition, since the discriminant analysis method can separate the pre-diabetes group well from the other two groups, it can lead to the development of new diagnostic tool for the earlier detection of the disease.

\section{Competing interests}

The authors declare that they have no competing interests.

\section{Authors' contributions}

YD and MQY conceived the project; $Y D$ and $C L$ designed and coordinated the study. CL, YL, SX, QK ZW, WY and MQY performed the experiments and analyzed the data. $L L, H W, X S, X Y$, and JYY participated in array data analyses. $\mathrm{CL}$ and $\mathrm{WY}$ summarized the results and drafted the manuscript. WY and $Y D$ revised and finalized the manuscript which was read and approved by all authors.

\section{Acknowledgements}

The research and publication of the research were supported by the Natural Science Foundation Hubei Province of China (2011CDB236 and 2012FFB04903), Open Research Fund Program of the State Key Laboratory of Virology of China (2012002) and United States National Institutes of Health (NIH). MQY was supported by NIH 5P20GM10342913 and ASTA Award \#15-B23, and YD was supported by $\mathrm{NIH}$ R21CA164764.

This article has been published as part of BMC Bioinformatics Volume 15 Supplement 17, 2014: Selected articles from the 2014 International Conference on Bioinformatics and Computational Biology. The full contents of the supplement are available online at http://www.biomedcentral.com/ bmcbioinformatics/supplements/15/S17.

\section{Authors' details \\ ${ }^{1}$ Wuhan University of Science and Technology, Wuhan, China. ${ }^{2}$ Rush University Cancer Center, and Departments of Internal Medicine and Biochemistry, Rush University Medical Center, Chicago, IL, USA. ${ }^{3}$ Guangxi Armed Police General Hospital, Nanning, Guangxi Autonomous Region, China. ${ }^{4}$ Department of Computer Science, George W. Donaghey College of Engineering and Information Technology, University of Arkansas at Little Rock, 2801 S. University Avenue, Little Rock, Arkansas 72204, USA. ${ }^{5}$ National 48610 Hospital of Armed Police Force, Panjin, Liaoning Province, China. ${ }^{6}$ Division of Biostatistics and Biomathematics, Massachusetts General Hospital and Harvard Medical School. Boston, MA 021 14, USA. ${ }^{7}$ Center for Computational Biology and Bioinformatics, Indiana University School of Medicine. Indiana University Purdue University Indianapolis, Indiana 46202, USA. ${ }^{8}$ MidSouth Bioinformatics Center, Department of Information Science, George W. Donaghey College of Engineering and Information Technology, University of Arkansas at Little Rock, 2801 S. University Avenue, Little Rock, Arkansas 72204, USA. ${ }^{9}$ Joint Bioinformatics Graduate Program, University of Arkansas at Little Rock and University of Arkansas for Medical Sciences, Little Rock, Arkansas 72204, USA.}

Published: 16 December 2014

\section{References}

1. World Health Organization. [http://www.who.int/diabetes/facts/ world_figures/en/index.html].
2. Centers for Disease Control and Prevention. [http://www.cdc.gov/ diabetes/pubs/pdf/ndfs_20].

3. From the Centers for Disease Control and Prevention. [http://www.cdc. $\mathrm{gov} / \mathrm{mmwr} /$ preview/M].

4. Letchuman GR, Wan NW, Wan MW, Chandran LR, Tee GH, Jamaiyah $H$, Isa MR, Zanariah H, Fatanah I, Ahmad FY: Prevalence of diabetes in the Malaysian National Health Morbidity Survey III 2006. Med J Malaysia 2010, 65:180-186.

5. Thorpe LE, Upadhyay UD, Chamany S, Garg R, Mandel-Ricci J, Kellerman S, Berger DK, Frieden TR, Gwynn C: Prevalence and control of diabetes and impaired fasting glucose in New York City. Diabetes Care 2009, 32:57-62.

6. Escobedo J, Buitron LV, Velasco MF, Ramirez JC, Hernandez R, Macchia A, Pellegrini F, Schargrodsky H, Boissonnet C, Champagne BM: High prevalence of diabetes and impaired fasting glucose in urban Latin America: the CARMELA Study. Diabet Med 2009, 26:864-871.

7. Qian Y, Lin Y, Zhang T, Bai J, Chen F, Zhang Y, Luo S, Shen H: The characteristics of impaired fasting glucose associated with obesity and dyslipidaemia in a Chinese population. BMC Public Health 2010, 10:139.

8. Herder C, Brunner EJ, Rathmann W, Strassburger K, Tabak AG, Schloot NC, Witte DR: Elevated levels of the anti-inflammatory interleukin-1 receptor antagonist precede the onset of type 2 diabetes: the Whitehall II study. Diabetes Care 2009, 32:421-423.

9. Herder C, Illig T, Rathmann W, Martin S, Haastert B, Muller-Scholze S, Holle R, Thorand B, Koenig W, Wichmann HE, Kolb H: Inflammation and type 2 diabetes: results from KORA Augsburg. Gesundheitswesen 2005, 67(Suppl 1):S115-S121.

10. Kong L, Zhu J, Han W, Jiang X, Xu M, Zhao Y, Dong Q, Pang Z, Guan Q, Gao $L$, et al: Significance of serum microRNAs in pre-diabetes and newly diagnosed type 2 diabetes: a clinical study. Acta Diabetol 2011, 48:61-69.

11. Frayling TM, Hattersley AT, McCarthy A, Holly J, Mitchell SM, Gloyn AL, Owen K, Davies D, Smith GD, Ben-Shlomo Y: A putative functional polymorphism in the IGF-I gene: association studies with type 2 diabetes, adult height, glucose tolerance, and fetal growth in U.K. populations. Diabetes 2002, 51:2313-2316.

12. Singh K, Agrawal NK, Gupta SK, Singh K: Association of Variant rs7903146 $(C / T)$ Single Nucleotide Polymorphism of TCF7L2 Gene With Impairment in Wound Healing Among North Indian Type 2 Diabetes Population: A Case-Control Study. Int I Low Extrem Wounds 2013.

13. Reichelt AJ, Spichler ER, Branchtein L, Nucci LB, Franco LJ, Schmidt Ml: Fasting plasma glucose is a useful test for the detection of gestational diabetes. Brazilian Study of Gestational Diabetes (EBDG) Working Group. Diabetes Care 1998, 21:1246-1249.

14. Ealovega MW, Tabaei BP, Brandle M, Burke R, Herman WH: Opportunistic screening for diabetes in routine clinical practice. Diabetes Care 2004, 27:9-12.

15. McCarthy MI, Zeggini E: Genome-wide association studies in type 2 diabetes. Curr Diab Rep 2009, 9:164-171.

16. Palmer CN: Novel insights into the etiology of diabetes from genomewide association studies. Diabetes 2009, 58:2444-2447.

17. Sladek R, Rocheleau G, Rung J, Dina C, Shen L, Serre D, Boutin P, Vincent D, Belisle A, Hadjadj $S$, et al: A genome-wide association study identifies novel risk loci for type 2 diabetes. Nature 2007, 445:881-885.

18. Frayling TM: Genome-wide association studies provide new insights into type 2 diabetes aetiology. Nat Rev Genet 2007, 8:657-662.

19. Zeggini E: A new era for Type 2 diabetes genetics. Diabet Med 2007, 24:1181-1186.

20. Zhao C, Mao J, Ai J, Shenwu M, Shi T, Zhang D, Wang X, Wang Y, Deng Y: Integrated lipidomics and transcriptomic analysis of peripheral blood reveals significantly enriched pathways in type 2 diabetes mellitus. $B M C$ Med Genomics 2013, 6(Suppl 1):S12.

21. Sakoda H, Ogihara T, Anai M, Funaki M, Inukai K, Katagiri H, Fukushima $Y$, Onishi $Y$, Ono H, Yazaki Y, et al: No correlation of plasma cell 1 overexpression with insulin resistance in diabetic rats and $3 \mathrm{T3}-\mathrm{L} 1$ adipocytes. Diabetes 1999, 48:1365-1371.

22. Yang YH, Dudoit S, Luu P, Lin DM, Peng V, Ngai J, Speed TP: Normalization for CDNA microarray data: a robust composite method addressing single and multiple slide systematic variation. Nucleic Acids Res 2002, 30:e15.

23. Grayson BL, Wang L, Aune TM: Peripheral blood gene expression profiles in metabolic syndrome, coronary artery disease and type 2 diabetes. Genes Immun 2011, 12:341-351. 
24. Wang-Sattler R, Yu Z, Herder C, Messias AC, Floegel A, He Y, Heim K, Campillos M, Holzapfel C, Thorand B, et al: Novel biomarkers for prediabetes identified by metabolomics. Mol Syst Biol 2012, 8:615.

25. Wang TJ, Larson MG, Vasan RS, Cheng $S$, Rhee EP, McCabe E, Lewis GD, Fox CS, Jacques PF, Fernandez C, et al: Metabolite profiles and the risk of developing diabetes. Nat Med 2011, 17:448-453.

doi:10.1186/1471-2105-15-S17-S5

Cite this article as: Liu et al:. Developing discriminate model and comparative analysis of differentially expressed genes and pathways for bloodstream samples of diabetes mellitus type 2. BMC Bioinformatics 2014 15(Suppl 17):S5.

Submit your next manuscript to BioMed Central and take full advantage of:

- Convenient online submission

- Thorough peer review

- No space constraints or color figure charges

- Immediate publication on acceptance

- Inclusion in PubMed, CAS, Scopus and Google Scholar

- Research which is freely available for redistribution

Submit your manuscript at www.biomedcentral.com/submit
C Biomed Central 